\title{
THE WEAK CONVERGENCE OF UNIT VECTORS TO ZERO IN HILBERT SPACE IS THE-CONVERGENCE OF ONE-DIMENSIONAL SUBSPACES IN THE ORDER TOPOLOGY
}

\author{
VLADIMIR PALKO
}

(Communicated by Dale Alspach)

\begin{abstract}
In this paper we deal with the (o)-convergence and the order topology in the hilbertian logic $\mathscr{L}(H)$ of closed subspaces of a separable Hilbert space $H$. We compare the order topology on $\mathscr{L}(H)$ with some other topologies. The main result is a theorem which asserts that the weak convergence of a sequence of unit vectors to zero in $H$ is equivalent to the convergence of the sequence of one-dimensional subspaces generated by these vectors to the zero subspace in the order topology on $\mathscr{L}(H)$.
\end{abstract}

\section{INTRODUCTION}

The notion of (o)-convergence was introduced by G. Birkhoff (see [B1], [B2]) and, independently, by Kantorovich $([\mathrm{K}])$. Let $\mathscr{L}$ be a quantum logic, i.e., an orthomodular lattice (for definition see [V]). For any $a \in \mathscr{L}$ we denote by $a^{\perp}$ the orthocomplement of $a$. We say that the net $a_{\alpha}$ of elements of $\mathscr{L}(o)$ converges to $a \in \mathscr{L}$ (written $a_{\alpha} \stackrel{(o)}{\longrightarrow} a$ ), if there exist nets $b_{\alpha}, c_{\alpha}$ such that $b_{\alpha} \leqq a_{\alpha} \leqq c_{\alpha}$ and $b_{\alpha} \nearrow a, c_{\alpha} \searrow a .\left(b_{\alpha} \nearrow a, c_{\alpha} \searrow a\right.$ means that $b_{\alpha}$ is increasing, $\bigvee b_{\alpha}=a$, and $c_{\alpha}$ is decreasing, $\wedge c_{\alpha}=a$.) The order topology $\tau_{o}$ on $\mathscr{L}$ is the strongest topology such that $(o)$-convergence of a net implies the topological convergence. If $\mathscr{L}$ is separable (i.e. every set of pairwise orthogonal elements of $\mathscr{L}$ is at most countable), then in the definition of the order topology it suffices to use sequences instead of nets (see [S]). In this case, $a_{n} \stackrel{(o)}{\longrightarrow} a$ is equivalent to the equality $\bigvee_{n=1}^{\infty} \bigwedge_{k=n}^{\infty} a_{k}=a=\bigwedge_{n=1}^{\infty} \bigvee_{k=n}^{\infty} a_{k}$. The order topology was studied very intensively in recent years from various points of view, for example in [E], [EW]. The comparison with other topologies on $\mathscr{L}$ was studied in [PR1], [PR2], [PR3], [R1], [R2]. Relatively few results are known about the order topology on the hilbertian logic $\mathscr{L}(H)$ of closed subspaces of a separable infinite-dimensional Hilbert space $H$. If $\operatorname{dim} H$ is finite, then $\tau_{0}$ is discrete on $\mathscr{L}(H)$. In most of the papers mentioned above there was used the assumption

Received by the editors June 16, 1993.

1991 Mathematics Subject Classification. Primary 06B30,46C05; Secondary 81P10.

Key words and phrases. Order topology, Hilbert space, quantum logic, weak convergence. 
of (o)-continuity of $L$ (it means that $x_{\alpha} \nearrow x$ implies $x_{\alpha} \wedge a \nearrow x \wedge a$ ), which is not satisfied on $\mathscr{L}(H)$ in the case of infinite dimension of $H$. This paper is a contribution to the study of order topology on $\mathscr{L}(H)$. In the following, the Hilbert space $H$ is assumed to be infinite-dimensional, real or complex.

\section{COMPARISON WITH OTHER TOPOLOGIES ON $\mathscr{L}(H)$}

Let us denote $[H]$ the space of all bounded operators on $H$. Some known topologies are defined on $[H]$, for example the uniform topology and the strong topology. A sequence of operators $A_{n}$ converges to operator $A$ in the uniform topology, if $\left\|A_{n}-A\right\| \rightarrow 0 . A_{n} \rightarrow A$ in the strong topology, if $\left\|A_{n} x-A x\right\| \rightarrow 0$ for every $x \in H$. Identifying the closed subspace $M \subset H$ with the orthogonal projector $P^{M}$, projecting on $M$, we can consider $\mathscr{L}(H)$ as a subset of $[H]$. Denote by $\tau_{\text {unif }}$ the restriction of the uniform topology from $[H]$ to $\mathscr{L}(H)$ and by $\tau_{\text {strong }}$ the restriction of the strong topology from $[H]$ to $\mathscr{L}(H)$. Then, for $M_{n}, M \in \mathscr{L}(H)$,

$$
\begin{gathered}
M_{n} \rightarrow M \text { in } \tau_{\text {unif }} \text { iff }\left\|P^{M_{n}}-P^{M}\right\| \rightarrow 0, \\
M_{n} \rightarrow M \text { in } \tau_{\text {strong }} \text { iff }\left\|P^{M_{n}} x-P^{M} x\right\| \rightarrow 0 \text { for every } x \in H .
\end{gathered}
$$

In [P] was proved that $\tau_{\text {strong }}$ is metrizable and $M_{n} \rightarrow M$ in $\tau_{\text {strong }}$ iff $\mu\left(M_{n}\right) \rightarrow \mu(M)$ for every $\sigma$-additive measure $\mu$ on $\mathscr{L}(H)$. In the following if $M, N \in \mathscr{L}(H), N \subset M$, then, for $M \wedge N^{\perp}$, we use the notation $M-N$. If $x \in H$ is nonzero, then $[x]$ denotes the one-dimensional subspace generated by $x$. For the zero vector and zero subspace we use the same symbol 0 .

Lemma 2.1. If $\left\|x_{n}-x\right\| \rightarrow 0, x \neq 0$, then $\left[x_{n}\right] \rightarrow[x]$ in $\tau_{\text {unif }}$ (and in $\tau_{\text {strong }}$ as well).

Proof. It is a simple exercise (see also [P], Lemma 4.1).

Lemma 2.2. If $M_{n}, M \in \mathscr{L}(H), M_{n} \stackrel{(o)}{\longrightarrow} M$, then $M_{n} \rightarrow M$ in $\tau_{\text {strong }}$.

Proof. Obviously, $M_{n} \nearrow M$ or $M_{n} \searrow M$ implies $M_{n} \rightarrow M$ in $\tau_{\text {strong. Let }}$ $M_{n}, M \in \mathscr{L}(H)$ be arbitrary, $M_{n} \stackrel{(o)}{\longrightarrow} M$. There exist $A_{n}, B_{n} \in \mathscr{L}(H)$, $A_{n} \subset M_{n} \subset B_{n}, A_{n} \nearrow M, B_{n} \searrow M$. We obtain

$$
\left\|P^{A_{n}} x-P^{M} x\right\| \rightarrow 0 \text { and }\left\|P^{M} x-P^{B_{n}} x\right\| \rightarrow 0
$$

for every $x \in H$. This implies $\left\|P^{B_{n}} x-P^{A_{n}} x\right\| \rightarrow 0$.

$$
\begin{aligned}
\left\|P^{B_{n}} x-P^{A_{n}} x\right\|^{2} & =\left\|P^{B_{n}-A_{n}} x\right\|^{2} \\
& =\left\|P^{B_{n}-M_{n}} x\right\|^{2}+\left\|P^{M_{n}-A_{n}} x\right\|^{2} \rightarrow 0 .
\end{aligned}
$$

Then, according to (1) and (2), we obtain

$$
\begin{aligned}
\left\|P^{M_{n}} x-P^{M} x\right\| & \leqq\left\|P^{M_{n}} x-P^{A_{n}} x\right\|+\left\|P^{A_{n}} x-P^{M} x\right\| \\
& =\left\|P^{M_{n}-A_{n}} x\right\|+\left\|P^{A_{n}} x-P^{M} x\right\| \rightarrow 0,
\end{aligned}
$$

so $M_{n} \rightarrow M$ in $\tau_{\text {strong }}$. The lemma is proved.

This lemma and the definition of the order topology imply immediately $\tau_{\text {strong }} \subset \tau_{o}$. The following two examples show that $\tau_{o}$ and $\tau_{\text {unif }}$ are not comparable. 
Example 2.3. Let $\varphi_{k=1}^{\infty}$ be an orthonormal system in $H$ and $M_{n}=\bigvee_{k=n}^{\infty}\left[\varphi_{k}\right]$, $n=1,2, \ldots . M_{n} \stackrel{(o)}{\longrightarrow} 0$ and, hence, $M_{n} \rightarrow 0$ in $\tau_{o}$, but $M_{n} \nrightarrow 0$ in $\tau_{\text {unif }}$, because 0 is isolated point in $\tau_{\text {unif }}$.

Example 2.4. Let $\varphi_{1}, \varphi_{2}$ be mutually orthogonal unit vectors and $\psi_{n}=\varphi_{1}+$ $\frac{1}{n} \varphi_{2}, n=1,2, \ldots$. Denote $B=\left\{\left[\psi_{n}\right] ; n=1,2, \ldots\right\}$. For any separable quantum logic $\mathscr{L}$, a subset $A \subset \mathscr{L}$ is closed in $\tau_{o}$ iff $a_{n} \in A, a_{n} \stackrel{(o)}{\longrightarrow} a$ implies $a \in A$ ([B2]). We shall show that $B$ is closed in $\tau_{o}$. Let $M_{n} \in B$ be a sequence, which is not constant starting from any $n$. Then $\bigvee_{n=1}^{\infty} \bigwedge_{k=n}^{\infty} M_{k}$ is the zero subspace 0 and $\bigwedge_{n=1}^{\infty} \bigvee_{k=n}^{\infty} M_{k}$ is the two-dimensional subspace containing $\varphi_{1}$ and $\varphi_{2}$. So, $M_{n}$ is not (o)-convergent. This implies that $B$ is closed in $\tau_{o}$ and, hence, $\left[\psi_{n}\right] \nrightarrow\left[\varphi_{1}\right]$ in $\tau_{o}$. However, $\left\|\psi_{n}-\varphi_{1}\right\| \rightarrow 0$ and, by Lemma $2.1,\left[\psi_{n}\right] \rightarrow\left[\varphi_{1}\right]$ in $\tau_{\text {unif }}$ (and also in $\tau_{\text {strong }}$ ).

Summarizing, we obtain

$$
\begin{aligned}
& \tau_{\text {strong }} \varsubsetneqq \tau_{\text {unif }}, \\
& \tau_{\text {strong }} \varsubsetneqq \tau_{o}, \\
& \tau_{\text {strong }} \subset \tau_{\text {unif }} \cap \tau_{o} .
\end{aligned}
$$

Open problem. $\tau_{\text {strong }}=\tau_{\text {unif }} \cap \tau_{o}$ ?

\section{THE WEAK CONVERGENCE OF VECTORS AS THE CONVERGENCE OF CORRESPONDING SUBSPACES IN THE ORDER TOPOLOGY}

A sequence $\varphi_{n}$ converges to $\varphi$ weakly in $H$, if the sequence of scalar products $\left(\varphi_{n}, u\right)$ converges to $(\varphi, u)$ for every $u \in H$.

Lemma 3.1. If $\varphi_{n} \in H,\left\|\varphi_{n}\right\|=1, n=1,2, \ldots$, then $\varphi_{n} \rightarrow 0$ weakly iff $\left[\varphi_{n}\right] \rightarrow 0$ in $\tau_{\text {strong. }}$.

Proof. It follows from the equality $\left|\left(u, \varphi_{n}\right)\right|=\left\|P^{\left[\varphi_{n}\right]} u\right\|, u \in H$.

Lemma 3.2. Let $f_{n}$ be an orthonormal system in $H$ and $g_{n} \in H,\left\|g_{n}\right\|=1$, $\left\|f_{n}-g_{n}\right\|<1 / 2^{n}, \bigvee_{i=1}^{n}\left[f_{i}\right]=\bigvee_{i=1}^{n}\left[g_{i}\right], n=1,2 \ldots$, and $\left|\left(g_{i}, g_{j}\right)\right|<1 / 2^{i+j}$, $i \neq j$. Then $\left[g_{n}\right] \stackrel{(o)}{\longrightarrow} 0$.

Proof. We have to show that $\bigwedge_{n=1}^{\infty} \bigvee_{i=n}^{\infty}\left[g_{i}\right]=0$. We shall prove it by contradiction. Let us assume that there exists $u \in H,\|u\|=1, u \in \bigvee_{i=n}^{\infty}\left[g_{i}\right]$ for every positive integer $n$. We shall show that this implies, for every integer $N>0$ and real $\varepsilon>0, \sum_{i=1}^{N}\left|\left(u, f_{i}\right)\right|^{2}<\varepsilon^{2}$, which is a contradiction to the equality $1=\|u\|^{2}=$ $\left\|\sum_{n=1}^{\infty}\left(u, f_{n}\right) f_{n}\right\|^{2}=\sum_{n=1}^{\infty}\left|\left(u, f_{n}\right)\right|^{2}$. Let an arbitrary integer $N>0$ and real $\varepsilon$, 
$0<\varepsilon<1$, be given. Let us choose integer $n_{0}$ such that $n_{0}>N, \frac{1}{2^{n_{0}-2}}<\frac{\varepsilon}{4}$ and

$$
\left\|u-\sum_{i=1}^{n_{0}}\left(u, f_{i}\right) f_{i}\right\|<\frac{\varepsilon}{4} .
$$

We have $u \in \bigvee_{i=n_{0}}^{\infty}\left[g_{i}\right]$. Then there exist constants $\lambda_{i}$ and integers $n_{i} \geqq n_{0}$, $i=1, \ldots, s$, such that

$$
\left\|u-\sum_{i=1}^{s} \lambda_{i} g_{n_{i}}\right\|<\frac{\varepsilon}{4}
$$

Since $\|u\|=1$, we obtain from (2)

$$
\left(1-\frac{\varepsilon}{4}\right)^{2} \leqq\left\|\sum_{i=1}^{s} \lambda_{i} g_{n_{i}}\right\|^{2} \leqq\left(1+\frac{\varepsilon}{4}\right)^{2} .
$$

Moreover,

$$
\left\|\sum_{i=1}^{s} \lambda_{i} g_{n_{i}}\right\|^{2}=\sum_{\substack{i, j=1 \\ i \neq j}}^{s} \lambda_{i} \bar{\lambda}_{j}\left(g_{n_{i}}, g_{n_{j}}\right)+\sum_{i=1}^{s}\left|\lambda_{i}\right|^{2} .
$$

Denote $d=\max \left\{\left|\lambda_{i}\right|^{2} ; i=1, \ldots, s\right\}$.

$$
\begin{aligned}
\sum_{\substack{i, j=1 \\
i \neq j}}^{s} \lambda_{i} \bar{\lambda}_{j}\left(g_{n_{i}}, g_{n_{j}}\right) & \geqq-\sum_{\substack{i, j=1 \\
i \neq j}}^{s}\left|\lambda_{i}\right|\left|\lambda_{j}\right| \mid\left(g_{n_{i}}, g_{n_{j}} \mid \geqq-d \sum_{\substack{i, j=1 \\
i \neq j}}^{s} \frac{1}{2^{n_{i}+n_{j}}}\right. \\
& \geqq-d \sum_{i=n_{0}}^{\infty} \sum_{j=n_{0}}^{\infty} \frac{1}{2^{i}} \frac{1}{2^{j}}=-\frac{d}{2^{2 n_{0}-2}} .
\end{aligned}
$$

Hence, by (4),

$$
\left\|\sum_{i=1}^{s} \lambda_{i} g_{n_{i}}\right\|^{2} \geqq d\left(1-\frac{1}{2^{2 n_{0}-2}}\right)+\left(\sum_{i=1}^{s}\left|\lambda_{i}\right|^{2}-d\right)
$$

The immediate consequence is $\max \left|\lambda_{i}\right|=\sqrt{d}<2$. In the opposite case,

$$
\left\|\sum_{i=1}^{s} \lambda_{i} g_{n_{i}}\right\|^{2} \geqq 4-\frac{1}{2^{2 n_{0}-4}}>\left(1+\frac{\varepsilon}{4}\right)^{2}
$$

which is a contradiction to (3). So, we have obtained

$$
\left\|\sum_{i=1}^{s} \lambda_{i} g_{n_{i}}-\sum_{i=1}^{s} \lambda_{i} f_{n_{i}}\right\|=\left\|\sum_{i=1}^{s} \lambda_{i}\left(g_{n_{i}}-f_{n_{i}}\right)\right\| \leqq 2 \sum_{i=n_{0}}^{\infty} \frac{1}{2^{i}}=\frac{1}{2^{n_{0}-2}}<\frac{\varepsilon}{4} .
$$

From (1), (2), and (5) we obtain

This implies

$$
\left\|\sum_{i=1}^{n_{0}}\left(u, f_{i}\right) f_{i}-\sum_{i=1}^{s} \lambda_{i} f_{n_{i}}\right\|<\varepsilon
$$

$$
\left\|\sum_{i=1}^{n_{0}}\left(u, f_{i}\right) f_{i}-\sum_{i=1}^{s} \lambda_{i} f_{n_{i}}\right\|^{2}=\sum_{i=1}^{n_{0}}\left|\left(u, f_{i}\right)\right|^{2}+\sum_{i=1}^{s}\left|\lambda_{i}\right|^{2}<\varepsilon^{2} .
$$

Hence, $\sum_{i=1}^{N}\left|\left(u, f_{i}\right)\right|^{2} \leqq \sum_{i=1}^{n_{0}}\left|\left(u, f_{i}\right)\right|^{2}<\varepsilon^{2}$, which is the promised contradiction. Lemma 3.2 is proved.

This lemma enables us to claim the following theorem: 
Theorem 3.3. If $M_{n} \in \mathscr{L}(H)$ are one-dimensional, $n=1,2, \ldots$, then $M_{n} \rightarrow$ 0 in $\tau_{\text {strong }}$ iff $M_{n} \rightarrow 0$ in $\tau_{0}$.

Proof. Since $\tau_{\text {strong }} \subset \tau_{0}$, the convergence in $\tau_{0}$ implies the convergence in $\tau_{\text {strong }}$. To show the opposite implication, it suffices to show that any sequence of one-dimensional subspaces converging to 0 in $\tau_{\text {strong }}$ contains a subsequence (o)-converging to 0 . Let $\left\|\varphi_{n}\right\|=1, n=1,2, \ldots,\left[\varphi_{n}\right] \rightarrow 0$ in $\tau_{\text {strong. }}$. We shall choose from $\left[\varphi_{n}\right]$ a subsequence $(o)$-converging to 0 . By Lemma $3.1, \varphi_{n} \rightarrow 0$ weakly. We shall prove that there exist a sequence $g_{n}$, which is a subsequence of $\varphi_{n}$ with property $\left|\left(g_{i}, g_{j}\right)\right|<1 / 2^{i+j}$, if $i \neq j$, and an orthonormal sequence $f_{n}$ with the property

$$
\bigvee_{i=1}^{n}\left[g_{i}\right]=\bigvee_{i=1}^{n}\left[f_{i}\right], \quad n=1,2, \ldots,\left\|g_{n}-f_{n}\right\|<1 / 2^{n}
$$

Sequences $f_{n}, g_{n}$ will be constructed by mathematical induction. We use the Gramm-Schmidt orthogonalization process, modified slightly so that the sequence is orthogonalized before it is completely defined. Put $g_{1}=f_{1}=\varphi_{1}$. By assumption of the weak convergence of $\varphi_{n}$ to 0 , we have $\left(\varphi_{n}, \varphi_{1}\right) \rightarrow 0$. We can choose $k_{2}$ such that $\left|\left(\varphi_{k_{2}}, \varphi_{1}\right)\right|<1 / 2^{3}$ and

$$
\left\|\varphi_{k_{2}}-\frac{\varphi_{k_{2}}-\left(\varphi_{k_{2}}, \varphi_{1}\right) \varphi_{1}}{\left\|\varphi_{k_{2}}-\left(\varphi_{k_{2}}, \varphi_{1}\right) \varphi_{1}\right\|}\right\|<\frac{1}{2^{2}} .
$$

We put $g_{2}=\varphi_{k_{2}}, f_{2}=\left(g_{2}-\left(g_{2}, f_{1}\right) f_{1}\right) /\left\|g_{2}-\left(g_{2}, f_{1}\right) f_{1}\right\|$. Then $\left\|g_{2}-f_{2}\right\|$ $<1 / 2^{2},\left|\left(g_{1}, g_{2}\right)\right|<1 / 2^{1+2}$ and $\left(f_{1}, f_{2}\right)=0$. If $f_{i}, g_{i}$ with demanded properties are defined for $i=1,2, \ldots, m$, then from the weak convergence of $\varphi_{n}$ to 0 there follows the existence of $k_{m+1}$ such that

$$
\left|\left(\varphi_{k_{m+1}}, \varphi_{k_{i}}\right)\right|<\frac{1}{2^{m+1+i}}, \quad i=1, \ldots, m
$$

and

$$
\left\|\varphi_{k_{m+1}}-\frac{\varphi_{k_{m+1}}-\sum_{i=1}^{m}\left(\varphi_{k_{m+1}}, f_{i}\right) f_{i}}{\left\|\varphi_{k_{m+1}}-\sum_{i=1}^{m}\left(\varphi_{k_{m+1}}, f_{i}\right) f_{i}\right\|}\right\|<\frac{1}{2^{m+1}} .
$$

We put $g_{m+1}=\varphi_{k_{m+1}}$,

$$
f_{m+1}=\frac{g_{m+1}-\sum_{i=1}^{m}\left(g_{m+1}, f_{i}\right) f_{i}}{\left\|g_{m+1}-\sum_{i=1}^{m}\left(g_{m+1}, f_{i}\right) f_{i}\right\|}
$$

Then $\left(f_{m+1}, f_{i}\right)=0, i=1, \ldots, m$. Obviously, $\bigvee_{i=1}^{n}\left[g_{i}\right]=\bigvee_{i=1}^{n}\left[f_{i}\right], n=$ $1,2, \ldots$, which follows from the properties of Gramm-Schmidt orthogonalization. This implies also $\bigvee_{i=1}^{\infty}\left[f_{i}\right]=\bigvee_{i=1}^{\infty}\left[g_{i}\right]$. Then, by Lemma 3.2, $\left[g_{n}\right] \stackrel{(o)}{\longrightarrow} 0$. The theorem is proved.

Since a one-dimensional subspace can be represented with a unit vector, according to Lemma 3.1, we can formulate the result of the previous theorem as it is expressed in the title of this article.

Theorem 3.4. A sequence $\varphi_{n}$ of unit vectors converges to 0 weakly in $H$ iff $\left[\varphi_{n}\right]$ converges to 0 in order topology in $\mathscr{L}(H)$. 
Let us remark that $\left[\varphi_{n}\right] \rightarrow 0$ in $\tau_{0}$ iff it converges to 0 in $\tau_{o} \cap \tau_{u n i f}$. Thus, the result of Theorem 3.3 agrees with the conjecture $\tau_{\text {strong }}=\tau_{o} \cap \tau_{\text {unif }}$. The convergence in $\tau_{0}$ and the $(o)$-convergence are not equivalent on an arbitrary quantum logic $\mathscr{L}$, in general. The same is true in the special case of $\mathscr{L}(H)$. We shall see it in the following example.

Example 3.4. Let $\varphi_{n}$ be a complete orthonormal system in $H$. We shall show that the sequence $\left[(1 / \sqrt{n}) \varphi_{1}+\varphi_{n+1}\right]$ does not $(o)$-converge to 0 . For $n=$ $1,2, \ldots$, denote by $M_{n}$ the subspace generated by vectors $\varphi_{2}, \ldots, \varphi_{n+1}$. For every $k=n+1, n+2, \ldots, \frac{1}{\sqrt{k}} \varphi_{1}+\varphi_{k+1} \in M_{n}^{\perp}$. We show that $M_{n}^{\perp}=$ $\bigvee_{k=n+1}^{\infty}\left[\frac{1}{\sqrt{k}} \varphi_{1}+\varphi_{k+1}\right]$. Obviously, $M_{n}^{\perp} \supset \bigvee_{k=n+1}^{\infty}\left[\frac{1}{\sqrt{k}} \varphi_{1}+\varphi_{k+1}\right]$. Let us assume that the opposite inclusion is not true. Then there exists $u \in M_{n}^{\perp}, u \neq 0$, $u \perp \frac{1}{\sqrt{k}} \varphi_{1}+\varphi_{k+1}$, for $k \geqq n+1$. We have $u=\left(u, \varphi_{1}\right) \varphi_{1}+\sum_{i=n+2}^{\infty}\left(u, \varphi_{i}\right) \varphi_{i}$. Then $0=\left(u, \frac{1}{\sqrt{k}} \varphi_{1}+\varphi_{k+1}\right)=\frac{1}{\sqrt{k}}\left(u, \varphi_{1}\right)+\left(u, \varphi_{k+1}\right)$. Hence, $\left(u, \varphi_{k+1}\right)=$ $-\frac{1}{\sqrt{k}}\left(u, \varphi_{1}\right)$. Since $u \neq 0$ implies $\left(u, \varphi_{1}\right) \neq 0$, we have obtained that the series of squares of $\left|\left(u, \varphi_{k+1}\right)\right|$ is not convergent. This is a contradiction. Thus, $M_{n}^{\perp}=\bigvee_{k=n+1}^{\infty}\left[\frac{1}{\sqrt{k}} \varphi_{1}+\varphi_{k+1}\right]$, and, hence,

$$
0 \neq\left[\varphi_{1}\right]=\bigwedge_{n=1}^{\infty} M_{n}^{\perp}=\bigwedge_{n=1}^{\infty} \bigvee_{k=n+1}^{\infty}\left[\frac{1}{\sqrt{k}} \varphi_{1}+\varphi_{k+1}\right]
$$

So, $\left[\frac{1}{\sqrt{k}} \varphi_{1}+\varphi_{k+1}\right]$ does not $(o)$-converge to 0 . However, it converges to 0 in $\tau_{0}$, because $\frac{1}{\sqrt{k}} \varphi_{1}+\varphi_{n+1} \rightarrow 0$ weakly.

\section{REFERENCES}

[B1] G. Birkhoff, On the structure of abstract algebras, Proc. Cambridge Philos. Soc. 31 (1935), 433-454.

[B2] L Lattice theory, Amer. Math. Soc., Providence, RI, 1948.

[E] M. Erné, Order topological lattices, Glasgow Math. J. 21 (1980), 57-68.

[EW] M. Erné and S. Weck, Order convergence in lattices, Rocky Mountain J. Math. 10 (1980), 805-818.

[K] L.V. Kantorovich, $O$ poluuporjadocennych linejnych prostranstvach $i$ ich primeneniach $k$ teorii linejnych operacij, Dokl. Akad. Nauk 4 (1935), 11-14.

[P] V. Palko, Topologies on quantum logics induced by measures, Math. Slovaca 39 (1989), 175-189.

[PR1] S. Pulmannová and Z. Riečanová, A topology on quantum logics, Proc. Amer. Math. Soc. 106 (1989), 891-897.

[PR2] - Modular almost orthogonal quantum logics, Internat. J. Theoret. Phys. 31 (1992), 881-888.

[PR3] _ Logics with separating sets of measures, Math. Slovaca 41 (1991), 167-177.

[R1] Z. Riečanová, Topologies in atomic quantum logics, Acta Univ. Carolin.-Math. Phys. 30 (1989), 143-148. 509-518. 
[S] T. A. Sarymsakov, S. A. Ajupov, Z. Chadžijev, and V. J. Čilin, Uporjadocennyje algebry, FAN, Tashkent, 1983. (Russian)

[V] V. S. Varadarajan, Geometry of quantum theory, Springer-Verlag, New York, 1985.

Department of Mathematics, Faculty of Electrical Engeneering, Slovak Technical UNIVERSITY, Ilkovicova 3, 81219 BratislaVA, SLOVAKIA

E-mail address: palko@kmat.elf.stuba.sk 